\title{
Medical Quiz: Single Best Answer (SBA)
}

(Birdem Med J 2014; 4(1): 57)

\section{Question 1:}

A 52-year-old man, who was previously well, presented with a 3 weeks history of increasing jaundice associated with pale stools, dark urine and itching. There was no associated pain or fever. He was not on any medication. Blood tests confirmed a cholestatic pattern of hyperbilirubinaemia with markedly raised alkaline phosphatase, moderate elevation of transaminases and normal serum albumin. Regarding the planning of his further investigation it is true that: (www.medicinecpd.co.uk)

A. Absence of pain excludes gallstone obstruction of the common bile duct (CBD)

B. Absence of bile duct dilatation on ultrasound scan (USG) makes it safe to immediately proceed to liver biopsy

C. Magnetic resonance cholangio-pancreatography (MRCP) is inferior to endoscopic retrograde cholangio-pancreatography (ERCP) in the investigation of extra-hepatic cholestasis where ultrasonography (USG) fails to show the cause

D. The role of ERCP is primarily to undertake therapeutic measures which might avoid surgical intervention

E. A prolonged prothrombin time due to extra-hepatic cholestasis requires a 5 day course of intravenous vitamin K (5mg) to correct it

\section{Question 2:}

A 56-year-old woman, who was a non-smoker, was referred to the cough clinic. She had had a troublesome cough for more than 6 years and was concerned by coughing bouts as she could not socialize as before. She explained that when talking on the phone she was interrupted by cough. She sometimes started coughing about 10 minutes after finishing her meals. There was no sputum and she did not cough during sleep, though the cough was worse as soon as she laid down in bed. She had a past history of heartburn and indigestion that had responded to the proton pump inhibitor (PPI) omeprazole $20 \mathrm{mg}$ o.d. As she often had intermittent breathlessness, she used a salbutamol inhaler which had no effect on her cough. There were no features of rhinitis or allergy. Physical examination was unremarkable. Chest x-ray and spirometry were normal. The most appropriate next step in her management would be: (www.medicinecpd.co.uk)

A. Cough suppressant agents

B. Full dose PPI plus ranitidine

C. Increase the does of inhaler

D. Laryngoscopy

E. Oesophageal pH studies

\section{Question 3:}

A-24-year-old woman gave a 5 weeks history of severe left temporal headaches which persisted for about 20 minutes, occurred 5 times in each 24 hours, both by day and by night. The left eyelid would droop and the left eye water profusely and become bloodshot. The best treatment would be: (www.medicinecpd.co.uk)
A. Oral lithium carbonate
B. Oral prednisolone
C. Subcutaneous sumatriptan
D. Oral verapamil
E. Nasal zolmitriptan

\section{Question 4:}

A previously well 24 years old woman attended the antenatal clinic in her first pregnancy. She was symptom free but a soft systolic murmur was heard in the second left intercostal space. Regarding further evaluation of the murmur it is true that: (www.medicinecpd.co.uk)

A. No further action need to be taken as innocent systolic murmurs occur in most pregnant women

B. Immediate referral to a cardiologist without delaying by further investigation is the best course of action

C. Pulmonary stenosis is excluded by the absence of an ejection click 
D. Narrowing of the splitting of the second heart sound on inspiration would be a normal finding

E. Fixed splitting of the second heart sound on inspiration requires an atrial septal defect (ASD) need to be excluded

\section{Question 5:}

A 74-year-old man had been found to have atrial fibrillation (AF) four years previously. He was otherwise well. He had been on warfarin since the outset and the INR had remained on target at 2.5 on a $5 \mathrm{mg}$ dose. He monitored INR every 3 months and at his last visit 2 months ago the INR again was 2.5. He now presented with haematemesis having just returned from a 2 week holiday abroad, where he had needed treatment with a 5 days course of amoxicillin for a chest infection. He was not shocked, INR was 5.2 and early gastroscopy was planned. Meanwhile, apart from stopping warfarin, the best management of the prolonged INR would be: (www.medicinecpd.co.uk)

A. Restart warfarin when INR has fallen back into the therapeutic range

B. Give vitamin K 2 mg orally and repeat INR in 24 hours

C. Give prothrombin complex concentrate (PCC) with vitamin $\mathrm{K} 2 \mathrm{mg}$ intravenously and repeat INR after 10 minutes

D. Give prothrombin complex concentrate (PCC) and repeat INR after 10 minutes

E. Give fresh frozen plasma (FFP) 